18

\title{
Оценки констант электрон-фононной связи графена с металлическими и неметаллическими подложками
}

\author{
() С.Ю. Давыдов \\ Физико-технический институт им. А.Ф. Иофффе РАН, \\ Санкт-Петербург, Россия \\ Санкт-Петербургский национальный исследовательский университет \\ информационных технологий, механики и оптики, \\ Санкт-Петербург, Россия \\ E-mail: Sergei_Davydov@mail.ru \\ (Поступила в Редакцию 19 сентября 2017 г.)
}

Рассмотрены два режима взаимодействия графен-подложка: слабая ван-дер-ваальсова связь и сильная ковалентная связь. В первом случае используется потенциал Леннарда-Джонса, во втором - метод связывающих орбиталей Харрисона. Для констант электрон-фононного взаимодействия получены аналитические выражения, содержащие только два параметра: энергию связи графена с подложкой $E_{B}$ и расстояние $d$ между ними. Расчеты констант выполнены для металлических, полупроводниковых и диэлектрических подложек.

DOI: 10.21883/FTT.2018.04.45698.267

\section{1. Введение}

Фононные и рамановские спектры графена и влияние на эти спектры электрон-фононного взаимодействия исследуются достаточно интенсивно [1-5]. Подавляющее число теоретических работ в этой области представляют собой расчеты, выполненные в рамках различных модификаций теории функционала плотности (DFT). Для фононного спектра свободного графена наряду с численными расчетами используются модели силовых констант [6-12]. Однако для эпитаксиального графена (ЭГ) существуют, насколько нам известно, лишь две модели. Это модель силовых констант Айзавы и др. [4,13] для графена на металлической подложке и модель двух связанных осцилляторов [14], в рамках которой было рассмотрено влияние $\mathrm{SiC}$ подложки на смещение и уширение рамановского G-пика ЭГ. Аналогичная ситуация имеет место и при исследовании электрон-фононного взаимодействия $[1,2,5]$ : большинство DFT-расчетов посвящено свободному графену, но даже при изучении ЭГ основное внимание уделяется влиянию электронфононной связи на колебания в плоскости графена, а колебания графена относительно подложки находятся на периферии исследований [15-18].

В работе [19] нами была предложена достаточно простая модель электрон-фононной связи графена с подложкой. В настоящей работе, оставаясь в рамках этой модели, мы представим различные схемы оценки электронфононных констант, соответствующих взаимодействию однослойного графена с переходными металлами, полупроводниками и диэлектриками. При этом цель работы - выразить эти константы через энергию связи $E_{B}$ графена с подложкой и равновесное расстояние $d$ между ними. Преимущество модельного подхода - возможность получения аналитических результатов, удобных для интерпретации экспериментальных данных.
В дальнейшем под слабой связью графен-подложка мы будем понимать ван-дер-ваальсово взаимодействие, характерное для квазисвободного графена. Сильной связью будем считать ковалентное взаимодействие атомов графена с атомами подложки, соответствующее буферному углеродному слою. В первом случае значения $E_{B}$ и $d$ берутся из эксперимента или DFT-расчетов других авторов, во втором - вычисляются.

\section{2. Металлическая подложка}

2.1. В а н-д е р-в а а ль с о в я с в я з ь. В настоящее время достаточно надежно установлено, что энергия связи $E_{B}$ (на атом) графена с подложками из переходных металлов лежит в интервале 30-200 meV; нижнему пределу соответствуют значения $d \approx 3.3 \AA$, верхнему $d \approx 2.1 \AA[20-26]$. Нижний предел, таким образом, сразу же можно отнести к слабой связи. С верхним пределом дело обстоит сложнее: здесь уже могут присутствовать и ван-дер-ваальсова, и ковалентная связи.

Для описания слабой связи атомов графена с атомами подложки будем использовать двухатомный потенциал Леннарда-Джонса:

$$
V_{L J}(z)=4 \varepsilon\left[(\sigma / z)^{12}-(\sigma / z)^{6}\right],
$$

где $\sigma$ - характерная длина, определяющая равновесное значение межатомного расстояния $z_{L J}=d_{L J}=\sqrt[6]{2} \sigma$ $\approx 1.12 \sigma, \varepsilon-$ энергия связи. Воспользуемся теперь теорией ван-дер-ваальсовы адгезии ЭГ $[27,28]$, согласно которой взаимодействие $U$ (на единицу площади) монослоя графена с плоской поверхностью подложки:

$$
U(z)=-(\gamma / 2)\left[3(d / z)^{3}-(d / z)^{9}\right],
$$

здесь $\gamma=(10 \pi / 9)\left(n_{s u b} n_{g} d^{3}\right) \varepsilon-$ энергия адгезии (на единицу площади); $n_{s u b}$ - объемная плотность атомов под- 
ложки; $n_{g}=S_{1}^{-1}-$ двумерная плотность атомов графена; $S_{1}=3 \sqrt{3} a_{0}^{2} / 4 \approx 2.62 \AA^{2}-$ площадь, приходящаяся на один атом графена; $a_{0}=1.42 \AA-$ расстояние между ближайшими соседями графена; $d=\sqrt[6]{2 / 5} \sigma \approx 0.8 \sigma-$ расстояние графен-подложка.

Обратимся к задаче об электрон-фононной связи графен-подложка $[19,29]$ (необходимые для дальнейшего формулы приведены в Приложении, п. 1). Исходя из выражения (2), для упругой константы $k=\left(\partial^{2} U \cdot S_{1} / \partial z^{2}\right)_{d}$, деформационной константы $w$, равной производной по $z$ от первого слагаемого потенциала $U \cdot S_{1}$, и энергии электрон-фононного взаимодействия $\lambda=w^{2} / k$ (см. п. 1 Приложения и $[19,29])$ получим:

$$
k=27 E_{B} / d^{2}, \quad w=9 E_{B} / 2 d, \quad \lambda=3 E_{B} / 4,
$$

где $E_{B}=\gamma S_{1}$. Следовательно, для вычисления упругой и электрон-фононных констант необходимо знать только две величины: энергию связи $E_{B}$ (на атом графена) и расстояние $d$. Для параметров $k, w, \lambda$ и $d$ справедливы соотношения

$$
w=k d / 6, \quad \lambda=k d^{2} / 36 .
$$

Так как $k=M \omega_{\perp}^{2}\left(\omega_{\perp}-\right.$ частота колебаний листа графена относительно неподвижной металлической подложки, $M$ - масса атома углерода), то, измерив значение $\omega_{\perp}$ и зная $d$, можно определить константы $w$ и $\lambda$.

Оценим теперь безразмерную константу электронфононной связи $\xi=\lambda / \Gamma$, где $\Gamma=\pi \rho_{m} V^{2}-$ полуширина квазиуровня адатома углерода, $\rho_{m}-$ плотность состояний металла, $V-$ матричный элемент связи графенподложка [19]. В соответствии с моделью Фриделя для $d$-металлов, положим $\rho_{m}=10 / W_{d}$, где $W_{d}-$ ширина $d$-зоны подложки [30] (для отсутствующего в [30] лютеция $W_{d}=7.8 \mathrm{eV}[31]$ ). Полагая $V=3 E_{B} / 2$ (см. Приложение, матричный элемент $V$ приравниваем к первому слагаемому потенциала $U-S_{1}$, соответствующему притяжению), получим значения $\Gamma$ и $\xi$.

Результаты расчета параметров для граней (111) некоторых $d$-металлов представлены в табл. 1 и 2 . $\mathrm{B}$ табл. 1 в качестве исходных величин $d$ и $E_{B}$ приняты значения, рассчитанные в работе [20] методом LDA (приближение локальной электронной плотности). ${ }^{1}$ Для Ir значения $d$ и $E_{B}$ взяты из работы [21]. В табл. 2 приведены значения $d$ и $E_{B}$, полученные из расчета в рамках метода vdW-DF2 $2^{\mathrm{C} 09 x}$ в работе [22]. Как следует из табл. 1 и 2, значительные различия имеют место для $\mathrm{Cu}, \mathrm{Ag} \mathrm{Au}$. C другой стороны, вычисленные в работах [20-22] значения $d$ для $\mathrm{Ni}$, $\mathrm{Pt}$ и $\mathrm{Ir}$ отлично согласуются с экспериментальными величинами 2.1 и $3.3 \AA$ соответственно (см. ссылки в работах [20-22]). Как правило, связь графена с подложками $\mathrm{Co}, \mathrm{Ni}, \mathrm{Ru}$, $\mathrm{Rh}, \mathrm{Pd}$ и $\operatorname{Re}$ определяется как сильная $[20,21,26]$. Мы же, как уже отмечалось, к сильной связи относим только ковалентную связь с энергией порядка $1 \mathrm{eV}$.

\footnotetext{
${ }^{1}$ Мы исключили из рассмотрения (в качестве исходных параметров) результаты работы [19], полученные методом vdW-DF, вследствие их обоснованной критики в работах [22,23].
}

Таблица 1. Квазисвободный графен (ван-дер-ваальсова связь) на гранях (111) $d$-металлов: исходные данные $\left(d\right.$ и $\left.E_{B}\right)$ взяты из работы [20] (для $\mathrm{Ir}-$ из [21]), и результаты расчета

\begin{tabular}{c|c|c|c|c|c|c|c|c}
\hline Параметры & $\mathrm{Co}$ & $\mathrm{Ni}$ & $\mathrm{Cu}$ & $\mathrm{Pd}$ & $\mathrm{Ag}$ & $\mathrm{Pt}$ & $\mathrm{Au}$ & $\mathrm{Ir}$ \\
\hline$d, \AA$ & 2.08 & 2.08 & 3.21 & 2.33 & 3.32 & 3.25 & 3.35 & 3.38 \\
$E_{B}, \mathrm{meV}$ & 175 & 123 & 35 & 79 & 45 & 33 & 31 & 50 \\
$k, \mathrm{eV} / \AA^{2}$ & 1.09 & 0.77 & 0.09 & 0.39 & 0.11 & 0.08 & 0.07 & 0.12 \\
$w, \mathrm{meV} / \AA$ & 379 & 266 & 49 & 153 & 61 & 46 & 42 & 67 \\
$\lambda, \mathrm{meV}$ & 131 & 92 & 26 & 59 & 34 & 25 & 23 & 38 \\
$W_{d}, \mathrm{eV}$ & 4.35 & 3.78 & 2.80 & 5.40 & 3.63 & 7.00 & 5.28 & 8.71 \\
$\Gamma, \mathrm{meV}$ & 497 & 283 & 31 & 82 & 39 & 11 & 13 & 20 \\
$\zeta$ & 0.26 & 0.33 & 0.85 & 0.73 & 0.86 & 2.25 & 1.81 & 1.85
\end{tabular}

Таблица 2. Квазисвободный графен (ван-дер-ваальсова связь) на гранях (111) $d$-металлов: исходные данные $\left(d\right.$ и $\left.E_{B}\right)$ взяты из работы [22], и результаты расчета

\begin{tabular}{c|c|c|c|c|c|c}
\hline Параметры & $\mathrm{Ni}$ & $\mathrm{Cu}$ & $\mathrm{Pd}$ & $\mathrm{Ag}$ & $\mathrm{Pt}$ & $\mathrm{Au}$ \\
\hline$d, \AA$ & 2.07 & 2.94 & 2.92 & 3.23 & 3.24 & 3.29 \\
$E_{B}, \mathrm{meV}$ & 141 & 62 & 72 & 53 & 68 & 59 \\
$k, \mathrm{eV} / \AA^{2}$ & 0.89 & 0.19 & 0.23 & 0.14 & 0.17 & 0.15 \\
$w, \mathrm{meV} / \AA$ & 307 & 95 & 111 & 74 & 94 & 81 \\
$\lambda, \mathrm{meV}$ & 106 & 47 & 54 & 40 & 51 & 44 \\
$\Gamma, \mathrm{meV}$ & 372 & 97 & 68 & 55 & 47 & 47 \\
$\zeta$ & 0.28 & 0.48 & 0.80 & 0.73 & 1.09 & 0.95
\end{tabular}

Анализируя полученные результаты, отметим, прежде всего, низкие значения упругих констант $k$ по сравнению с константой центрального взаимодействия свободного графена $k_{0} \approx 22 \mathrm{eV} / \AA^{2}$ [32]. Низки и частоты нормальных колебаний слоя графена относительно подложки $\omega_{\perp}=\sqrt{k / M}$. Так, например, для подложки из кобальта частота $\omega_{\perp} \approx 19(18) \mathrm{meV}$, а для подложки из золота $\omega_{\perp} \approx 5(7) \mathrm{meV}$ при расчете по данным работ $[20]([21])$. Добавим сюда также результаты работы [33] по ЭГ на $\operatorname{Ru}(0001)$, согласно которым $d \approx 2.1 \AA$ и $\omega_{\perp} \approx 16 \mathrm{meV}$, что практически совпадает с результатами для подложки из кобальта. Для сравнения, при колебаниях в плоскости свободного графена частота длинноволновых оптических фононов $\omega_{\|} \approx 0.2 \mathrm{eV}$ (энергия $\mathrm{G}$-пика), для изгибных колебаний $\omega_{z} \approx 0.1 \mathrm{eV}[1,2,5]$. Такой результат естественен, так как взаимодействие в плоскости графена является ковалентным и $a_{0}$ значительно меньше $d$.

Далее, для ЭГ на $\mathrm{Ni}(111)$ наше значение $k$ в 2 и 5 раз меньше, чем силовые константы модели Айзавы $\alpha_{s}=2.21$ и $5.09 \mathrm{eV} / \AA^{2}$, полученные путем сравнения с численными расчетами по схемам GGA (generalized gradient approximation) и LDA (local density approximation), соответственно [34,35]. По данным работы [13], $\alpha_{s} \approx 0.19-0.31 \mathrm{eV} / \AA^{2}$, что значительно меньше полученного нами значения. И, наконец, в работах $[5,34]$ для $\mathrm{Ni}(111)$ приводится $\alpha_{s} \approx 0.65 \mathrm{eV} / \AA^{2}$, а для $\mathrm{Pt}(111)$ 

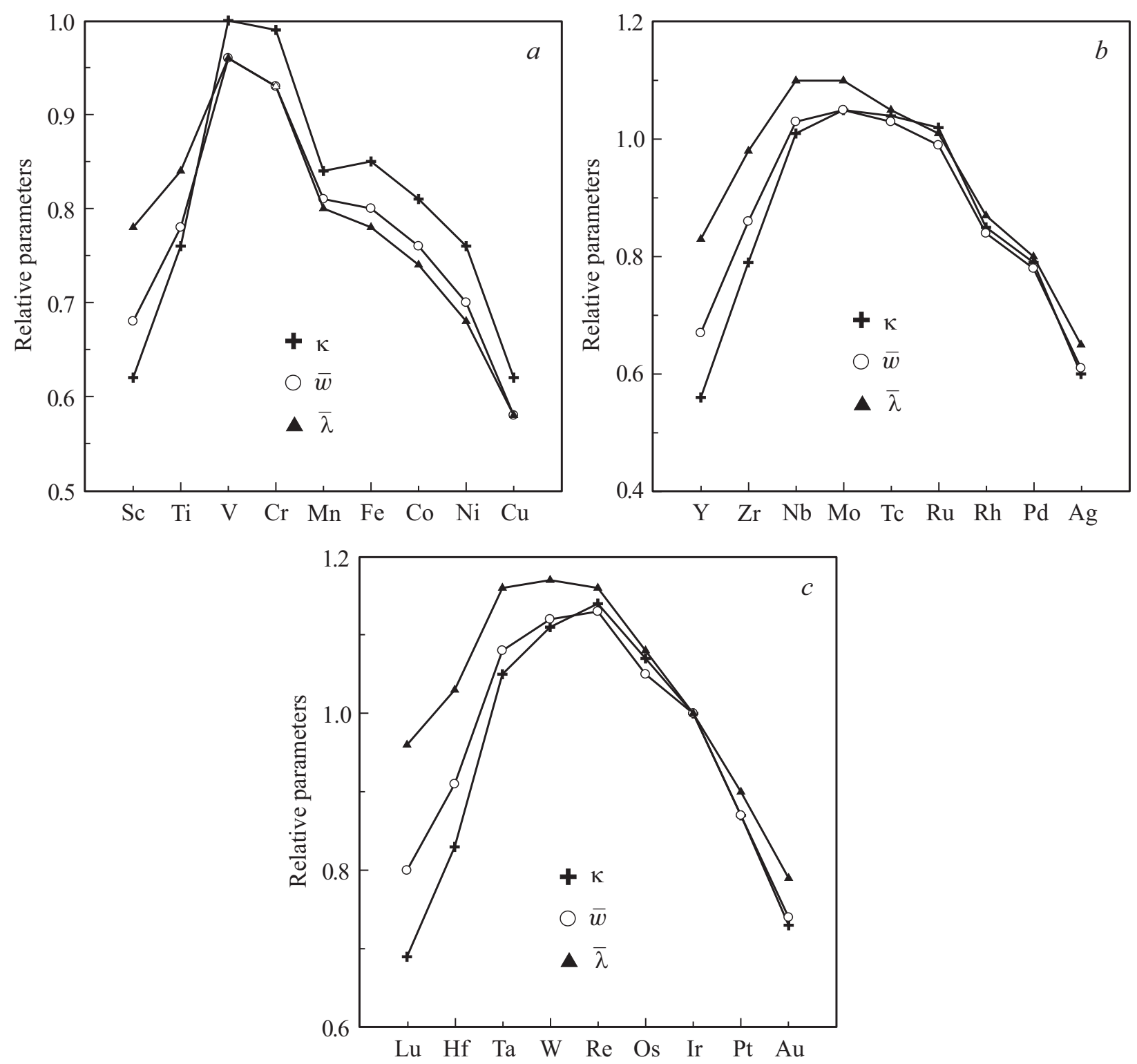

Рис. 1. Относительные параметры $\kappa=k(\mathrm{M}) / k(\mathrm{Ir}), \bar{w}=w(\mathrm{M}) / w(\mathrm{Ir}), \bar{\lambda}=\lambda(\mathrm{M}) / \lambda(\mathrm{Ir})$ для подложек $3 d$ - $(a), 4 d$ - $(b)$ и $5 d$-рядов $(c)$, вычисленные из соотношений (9).

нулевое значение $\alpha_{s}$ [5]. Оба последних результата близки к полученным нами значениям $k$.

Перейдем теперь к электрон-фононным константам. Для свободного графена $w_{\|} 2 t / a_{0} \approx 4 \mathrm{eV} / \AA$, где энергия перехода между ближайшими соседями в графене $t \approx 3 \mathrm{eV}$. Отсюда получаем $\lambda_{\|}=w_{\|}^{2} / M \omega_{\|}^{2} \approx 130 \mathrm{meV}$, что совпадает с нашим результатом для ЭГ на кобальте. Значения безразмерной константы электронфононного взаимодействия $\xi$ (табл. 1,2 ) лежат в интервале $0.26-2.25$. Этими оценками приходится, к сожалению, ограничиться.

2.2. Ковалентная связь. Пусть переходный металл характеризуется состоянием $|d\rangle$ с энергией $E_{d}$. Считаем, что связь атома углерода графена с атомом подложки осуществляется $p_{z}$-орбиталью с энергией $E_{p}$. По данным работы [36], матричный элемент взаимодей- ствия $d$ - и $p_{z}$-орбиталей (ковалентная энергия) примем равным

$$
V_{2}=V_{p d \sigma}=(3 \sqrt{15} / 2 \pi) \hbar^{2}\left(r_{d}^{3} r_{p}\right)^{12} / m_{0} d^{4},
$$

где $r_{p(d)}$ - радиус $p(d)$-состояния, $m_{0}$ - масса свободного электрона, $\hbar$ - приведенная постоянная Планка. Здесь и в дальнейшем используются обозначения метода связывающих орбиталей (МСО) Харрисона [37]. Пренебрегая полярностью связи графен-металл (см. Приложение, п. 2), определим энергию двухэлектронной связи атома подложки и углерода графена как

$$
E_{b}=2\left(-V_{2}+V_{\text {rep }}\right)
$$

где $V_{r e p}=A_{r e p}\left(r_{d} / d\right)^{8} \quad[18,19]$. Константу $A_{\text {rep }}$ найдем из условия равновесия $\left(\partial E_{d} / \partial d\right)_{0}=0$, так что 

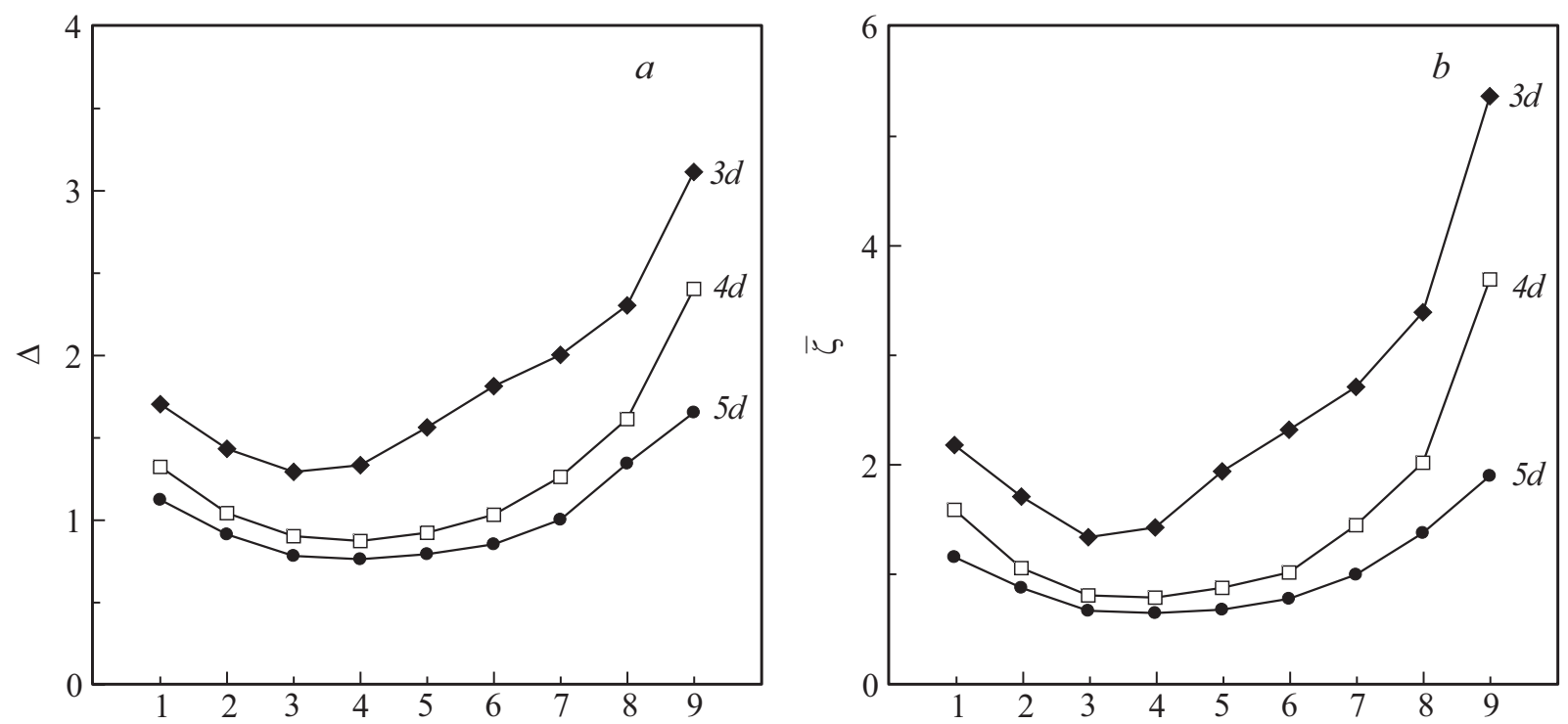

Рис. 2. Относительные параметры $\Delta=\left[W_{d}(\mathrm{Ir}) / W_{d}(\mathrm{M})\right](a)$ и $\bar{\xi}=\xi(\mathrm{M}) / \xi(\mathrm{Ir})=\Delta / \bar{\lambda}(b)$ для подложек рядов $3 d$, $4 d$ и $5 d$. Номера на горизонтальной оси соответствуют элементам $d$-рядов (рис. 1).

$A_{\text {rep }}=\left(V_{2} / 2\right)\left(d / r_{d}\right)^{8}$ и $E_{b}=-V_{2}$. Тогда, согласно п. 1 Приложения, вместо уравнения (3) получим

$$
k_{b}=32\left|E_{b}\right| / d^{2}, \quad w_{b}=4\left|E_{b}\right| / d, \quad \lambda_{b}=\left|E_{b}\right| / 2,
$$

где индекс $b$ означает, что данный параметр характеризует двухатомную ковалентную связь. Вместо уравнения (4) имеем:

$$
w_{b}=k_{b} d / 8, \quad \lambda_{b}=k_{b} d^{2} / 64 .
$$

Из формул (7) вытекают масштабные зависимости:

$$
k_{b} \propto r_{d}^{3 / 2} / d^{6}, \quad w_{b} \propto r_{d}^{3 / 2} / d^{5}, \quad \lambda_{b} \propto r_{d}^{3 / 2} / d^{4},
$$

позволяющие простым образом оценить влияние подложки на упругие и электрон-фононные характеристики ЭГ. В соответствии с МСО [37], для ковалентной связи $d=d_{c o v}=r_{\mathrm{C}}+r_{\mathrm{M}}$, где $r_{\mathrm{C}}=0.77 \AA-$ атомный радиус углерода, $r_{\mathrm{M}}-$ атомный радиус металла [38].

На рис. 1 представлены относительные параметры $\kappa=k_{b}(\mathrm{M}) / k_{b}(\mathrm{Ir}), \bar{w}=w_{b}(\mathrm{M}) / w_{b}(\mathrm{Ir})$ и $\bar{\lambda}=\lambda_{b}(\mathrm{M}) / \lambda_{b}(\mathrm{Ir})$ для металлов $3 d$-, $4 d$ - и $5 d$-рядов $\left[\bar{\lambda}_{b}=V_{2}(\mathrm{M}) / V_{2}(\mathrm{Ir})=\right.$ $\left.=E_{b}(\mathrm{M}) / E_{b}(\mathrm{Ir}), \sqrt{\kappa}=\omega_{\perp}(\mathrm{M}) / \omega_{\perp}(\mathrm{Ir})\right]$. При этом для иридия $E_{b} \approx-2.0 \mathrm{eV}, \quad k_{b} \approx 14 \mathrm{eV} / \AA^{2}, \quad w_{b} \approx 3.8 \mathrm{eV} / \AA$, $\lambda_{b} \approx 1.0 \mathrm{eV}$. Из рис. 1 видно, что все параметры минимальны для элементов начала и конца $d$-рядов и максимальны для центральных элементов $d$-рядов, для которых $\kappa \approx \bar{w} \approx \bar{\lambda} \approx 1$. Последний результат связан $\mathrm{c}$ тем обстоятельством, что значения $d$ и $r_{d}$ убывают от начала к концу $d$-ряда. Рис. 2 демонстрирует изменение параметров $\Delta=\left[W_{d}(\mathrm{Ir}) / W_{d}(\mathbf{M})\right]$ и $\bar{\xi}=\xi(\mathbf{M}) / \xi(\mathrm{Ir})=\Delta / \bar{\lambda}$ в рядах $d$-металлов. Видно, что зависимость $\bar{\xi}$ от номера $N$ элемента в $d$-ряду (рис. $2, b)$ качественно совпадает с зависимостями $\Delta(N)$ (рис. $2, a)$ и $\bar{\lambda}^{-1}(N)$. Таким образом, параметр $\bar{\xi}$ максимален для элементов начала и конца $d$-рядов.
Для перехода от энергии двухатомной связи $E_{b}$ к энергии связи графен-субстрат $E_{b}$ учтем, что только половина атомов графена ковалентно связана с подложкой (см. рис. 13 в [5] и рис. 1 в [34] для графена на $\mathrm{Ni}$ - модель Алларда-Виртца). Тогда $E_{b}=\left|E_{b}\right| / 2$, откуда вместо уравнений (7) и (8) получим:

$$
\begin{gathered}
k=32 E_{B} / d^{2}, \quad w=4 E_{B} / d, \quad \lambda=E_{B} / 2, \\
w=k d / 8, \quad \lambda=k d^{2} / 64 .
\end{gathered}
$$

Результаты расчета приведены в табл. 3 .

Из табл. 3 следует, что для рассмотренных металлов среднее расстояние графен-подложка $d \approx 2.1 \AA$, что практически совпадает с результатами для Со и $\mathrm{Ni}$ (табл. 1,2). На этом, однако, сходство результатов заканчивается. Полученные для ковалентной связи значения $E_{B}, k, w$ и $\lambda$ значительно превосходят ван-дерваальсовы величины, тогда как для $\xi$ имеем обратное соотношение. В работе [39] для ЭГ на $\mathrm{Cu}$ и $\mathrm{Ni}$ приводятся (чрезвычайно высокие, на наш взгляд) значения энергии адгезии $\gamma=0.80$ и $4.54 \mathrm{eV} / \AA^{2}$, откуда получаем $E_{B}=\gamma S_{1}=2.1$ и $11.9 \mathrm{eV}$. Напомним, что сильная ковалентная связь характерна для буферного углеродного слоя.

Таблица 3. Буферный слой (ковалентная связь): результаты расчета по Харрисону для металлических подложек

\begin{tabular}{c|c|c|c|c|c|c|c|c}
\hline Параметры & $\mathrm{Co}$ & $\mathrm{Ni}$ & $\mathrm{Cu}$ & $\mathrm{Pd}$ & $\mathrm{Ag}$ & $\mathrm{Pt}$ & $\mathrm{Au}$ & $\mathrm{Ir}$ \\
\hline$d, \AA$ & 2.02 & 2.01 & 2.05 & 2.14 & 2.21 & 2.16 & 2.21 & 2.13 \\
$E_{B}, \mathrm{eV}$ & 0.74 & 0.68 & 0.58 & 0.80 & 0.65 & 0.90 & 0.87 & 1.0 \\
$k, \mathrm{eV} / \AA^{2}$ & 5.8 & 5.4 & 4.4 & 5.6 & 4.3 & 6.2 & 5.7 & 7.1 \\
$w, \mathrm{eV} / \AA$ & 1.47 & 1.35 & 1.13 & 1.50 & 1.18 & 1.67 & 1.57 & 1.88 \\
$\lambda, \mathrm{eV}$ & 0.37 & 0.34 & 0.29 & 0.40 & 0.32 & 0.45 & 0.44 & 0.26 \\
$\Gamma, \mathrm{eV}$ & 15.8 & 15.4 & 15.1 & 14.9 & 14.6 & 14.5 & 18.0 & 14.4 \\
$\xi \cdot 10^{2}$ & 2.3 & 2.2 & 1.9 & 2.7 & 2.2 & 3.1 & 2.4 & 6.9
\end{tabular}


Таблица 4. Буферный слой $[\mathrm{SiC}(0001)]$ и квазисвободный графен $[\operatorname{SiC}(000 \overline{1}), \operatorname{Si}(100)]:$ исходные данные $\left(d\right.$ и $\left.E_{B}\right)$ взяты из работ [40-48], и результаты расчета для полупроводниковых подложек

\begin{tabular}{c|c|c|c}
\hline Параметры & $\begin{array}{c}\mathrm{SiC}(0001), \\
\text { ковалентная } \\
\text { связь }\end{array}$ & $\begin{array}{c}\mathrm{SiC}(000 \overline{1}), \\
\text { ван-дер-ваальсова } \\
\text { связь }\end{array}$ & $\begin{array}{c}\mathrm{Si}(100), \\
\text { ван-дер-ваальсова } \\
\text { связь }\end{array}$ \\
\hline$d, \AA$ & 1.95 & 3.35 & 3.00 \\
$E_{B}, \mathrm{meV}$ & 840 & 30 & 40 \\
$k, \mathrm{meV} / \AA^{2}$ & $3.98 \cdot 10^{3}$ & 72 & 119 \\
$w, \mathrm{meV} / \mathrm{A}$ & $3.88 \cdot 10^{3}$ & 40 & 59 \\
$\lambda, \mathrm{meV}$ & $3.78 \cdot 10^{3}$ & 23 & 30 \\
$\Gamma, \mathrm{meV}$ & $11.58 \cdot 10^{3}$ & 1.6 & 3.8 \\
$\xi$ & 0.33 & 14 & 8
\end{tabular}

\section{3. Неметаллические подложки}

3.1. $\mathrm{SiC}\{0001\}$-подлложка. В настоящее время считается установленным, что графеноподобный буферный слой образуется только на $\mathrm{SiC}(0001)$, т.е. на $\mathrm{Si}$ грани [40]. В этом случае связь графена с $\operatorname{SiC}(0001)$ является сильной (ковалентной): $E_{B} \approx 0.33-0.65 \AA \mathrm{eV}[41]$, $E_{B} \approx 0.36$ [42]. При этом $d \approx 2.0 \AA[42,43]$, что практически совпадает с суммой атомных радиусов кремния $r_{\mathrm{Si}}=1.18 \AA$ и углерода $r_{\mathrm{C}}=0.77 \AA$ [38] (в работе [44] $d \approx 1.62 \AA)$. Таким образом, значения $d$ и $E_{B}$ соответствуют ковалентной связи. Поэтому для дальнейших оценок воспользуемся МСО Харрисона [37].

Поверхностный атом полупроводниковой подложки характеризуется $s p^{3}$-орбиталью, а атом углерода графена $-p_{z}$-орбиталью. По данным работы [45], матричный элемент взаимодействия $s p^{3}$ - и $p_{z}$-орбиталей (ковалентная энергия) равен

$$
V_{2}=V_{p / s p^{3}}=1.26\left(\hbar^{2} / m_{0} d^{2}\right)
$$

Пренебрегая полярностью связи (см. Приложение, п. 3), легко показать [32], что энергия двухэлектронной связи атома графена с атомом подложки равна

$$
E_{b}=-2 V_{2} / 3
$$

а силовая константа центрального взаимодействия

$$
k=4 V_{2} / d^{2} .
$$

Деформационная константа электрон-фононного взаимодействия $w=-\partial V_{2} / \partial d$ равна

$$
w=2 V_{2} / d .
$$

Так что энергия электрон-фононного взаимодействия $\lambda=w^{2} / k$ имеет вид

$$
\lambda=V_{2}
$$

Для перехода от энергии двухатомной связи $E_{b}$ к энергии связи графен-субстрат $E_{B}$ учтем, что лишь треть атомов углерода графена ковалентно связана с атомами $\mathrm{SiC}$-подложки [40], так что $E_{B}=\left|E_{b}\right| / 3$ и

$$
\begin{gathered}
k=18 E_{B} / d^{2}, \quad w=9 E_{B} / d, \quad \lambda=9 E_{B} / 2, \\
w=k d / 2, \quad \lambda=k d^{2} / 4 .
\end{gathered}
$$

Полагая $\quad d=r_{\mathrm{C}}+r_{\mathrm{Si}}=1.95 \AA \quad[38], \quad$ получаем $V_{2} \approx 2.52 \mathrm{eV}$ и $E_{B} \approx 0.84 \mathrm{eV}$. Для оценки параметра $\xi$ нужно знать значение $\Gamma=\pi \rho_{s c} V^{2}$, где $\rho_{s c}=$ const плотность зонных состояний карбида кремния. Положим $\rho_{s c}=4 / W_{V B}$, где $W_{V B}-$ ширина валентной зоны. Так как $W_{V B} \approx 15.5 \mathrm{eV}[46]$, имеем $\Gamma=\pi \rho_{s c} V^{2} \approx 5.15 \mathrm{eV}$. Результаты оценок представлены в табл. 4. Сравнение полученных значений с результатами для буферного слоя на металлической подложке (табл. 3) показывает, что для $\mathrm{SiC}$ подложки $E_{B}$ имеет тот же порядок, $k$ в $\sim 1.5$ раза меньше, $w$ в $\sim 3$ раза больше, $\lambda$ приблизительно на порядок выше, а $\xi-$ более чем на два порядка.

Перейдем теперь к $\mathrm{SiC}(000 \overline{1})$, т.е. к ЭГ на $\mathrm{C}$ грани. Здесь буферный слой не образуется [40], хотя в ранних теоретических работах [42,43] такая структура углеродного слоя рассматривалась. Нам, к сожалению, неизвестны ни экспериментальные, ни расчетные энергии связи $E_{B}$ квазисвободного графена с С-гранью карбида кремния. Для полуколичественных оценок воспользуемся косвенными данными. Так, согласно данным работы [47], энергия адгезии графена на графите $\gamma=0.221 \mathrm{~J} / \mathrm{m}^{2}$, что практически совпадает с энергией межплоскостной адгезии графита $0.227 \mathrm{~J} / \mathrm{m}^{2}$ $\left(E_{B} \approx 30 \mathrm{meV}\right)$. Поэтому примем $d$ равным межплоскостному расстоянию в графите $3.35 \AA^{2}{ }^{2}$ В подтверждение такого выбора отметим, что для $\mathrm{Si}-г$ рани слой квазисвободного графена отстоит от буферного слоя на расстоянии 3.34-3.71 А [44]. Результаты оценок представлены в табл. 4.

В работе [48] показано, что энергия связи графена с гранью (100) кремниевой подложки $E_{B} \approx 40 \pm 7 \mathrm{meV}$, но значение $d$ не указано. Считая $d=3 \AA$, что типично для

\footnotetext{
${ }^{2}$ При этом, строго говоря, мы сводим задачу об ЭГ на С-грани карбида кремния к задаче об ЭГ на графите.
} 
Таблица 5. Квазисвободный графен (ван-дер-ваальсова связь): исходные данные $\left(d\right.$ и $\left.E_{B}\right)$ взяты из работ [49-52], и результаты расчета для диэлектрических подложек

\begin{tabular}{c|c|c|c|c}
\hline Параметры & $\mathrm{SnO}_{2}$ & $\mathrm{SiO}_{2}$ & $\mathrm{HfO}_{2}$ & $\mathrm{~h}-\mathrm{BN}$ \\
\hline$d, \AA$ & 3.00 & 3.07 & 3.05 & 3.35 \\
$E_{B}, \mathrm{meV}$ & 70 & 51 & 112 & 83 \\
$k, \mathrm{eV} / \AA^{2}$ & 0.21 & 0.15 & 0.24 & 0.20 \\
$w, \mathrm{meV} / \AA$ & 105 & 75 & 162 & 111 \\
$\lambda, \mathrm{meV}$ & 53 & 38 & 83 & 62 \\
$W_{V B}, \mathrm{eV}$ & 9 & 10 & 5 & 10 \\
$\Gamma, \mathrm{meV}$ & 23 & 11 & 106 & 19 \\
$\xi$ & 2.30 & 3.44 & 0.78 & 3.20
\end{tabular}

ван-дер-ваальсовой связи, получим значения $k, w$ и $\lambda$ (табл. 4). Принимая $W_{V B} \approx 12 \mathrm{eV}$ [37] и $\rho_{s c}=4 / W_{V B}$, получим значение $\xi$ (табл. 4).

3.2. Диэлектрические подложки. Рассмотрим достаточно широко используемые оксидные подложки $\mathrm{SnO}_{2}$ [49] и $\mathrm{SiO}_{2}$ [50], включая также $\mathrm{HfO}_{2}$ [51] (верхний слой подложки образуют атомы кислорода), и гексагональный нитрид бора h-BN [52]. По данным работ [50-52] и ссылок в них, значительную, а зачастую и доминирующую роль в связи графен-диэлектрик играет ван-дер-ваальсово взаимодействие. Действительно, во всех рассмотренных случаях $d \approx 3 \AA, E_{B} \leq 110 \mathrm{meV}$ $\left(=E_{B}\right.$ для ЭГ на $\left.\mathrm{HfO}_{2}\right)$. Поэтому для оценок констант воспользуемся формулами (3). Сравнение результатов расчета констант $k, w$ и $\lambda$ (табл. 5) с соответствующими константами в табл. 1 и 2 показывает, что полученные для $\mathrm{SnO}_{2}, \mathrm{SiO}_{2}$ и h-BN величины близки к приведенным в табл. 2 значениям для $\mathrm{Pd}, \mathrm{Ag}, \mathrm{Pt}$ и $\mathrm{Au}$. Несколько особняком стоит $\mathrm{HfO}_{2}$ : при $d \approx 3 \AA$ энергия связи $E_{B} \approx 112 \mathrm{meV}$, т. е. того же порядка, что и для $\mathrm{Ni}$ $(141 \mathrm{meV})$, имеющего $d \approx 2 \AA$. Предполагается поэтому, что в случае ЭГ на $\mathrm{HfO}_{2}$ в дополнение к ван-дерваальсовой связи имеет место гибридизация состояний $p_{z}$ атомов С графена и $2 p$-состояний кислорода подложки. Мы, однако, на этом вопросе здесь останавливаться не будем.

Перейдем к оценкам безразмерной константы $\zeta$. Для ЭГ на оксидах положим $\rho_{\text {diel }}=6 / W_{V B}$, так что параметр $\Gamma=\pi \rho_{\text {diel }} V^{2}=27 \pi E_{B}^{2} / 2 W_{V B}$, где $W_{V B} \approx 9 \mathrm{eV}$ для $\mathrm{SnO}_{2}$ [53], $W_{V B} \approx 10 \mathrm{eV}$ для $\mathrm{SiO}_{2}{ }^{3}[37,54], W_{V B} \approx 5 \mathrm{eV}$ для $\mathrm{HfO}_{2}$ [55]. Для h-BN $\rho_{\text {diel }}=4 / W_{V B}$, так что $\Gamma=\pi \rho_{\text {diel }} V^{2}=9 \pi E_{B}^{2} / W_{V B}$ и $W_{V B} \approx 10 \mathrm{eV}[56]$. Оценки $\zeta$ приведены в табл. 5 .

\section{4. Заключительные замечания}

В работе [19] для изолированного углеродного адатома показано, что при $\xi<\pi$ отсутствует возможность скачкообразного изменения перехода заряда между изолированным адатомом и подложкой. Тут следует, однако,

\footnotetext{
3 Здесь мы игнорируем наличие узкой щели в валентной зоне, сформированной $p$-состояниями кислорода подложки.
}

оговориться. Если разрешить электронам переходить не только с адатома на металл, но и с металла на адатом, то плотность состояний (9) в работе [19] нужно умножить на 2. В результате вместо уравнения (24) в работе [19] получим $\operatorname{ctg}(\pi \bar{n} / 2)=\mathrm{const}-\xi \bar{n}$. Тогда критерий возможности скачкообразного изменения числа заполнения адатома $\bar{n}$ принимает вид $\xi>\pi / 2$. Этому неравенству удовлетворяют ван-дер-ваальсовы связи ЭГ с гранями (111) металлов Pt, $\mathrm{Au}$ и $\mathrm{Ir}$ (табл. 1), $\mathrm{SiC}(000 \overline{1})$ и $\mathrm{Si}$ (100) (табл. 4), $\mathrm{SnO}_{2}$ и h-BN (табл. 5).

По поводу оценок параметра $\zeta$ необходимо, однако, отметить следующее. В теории адсорбции полуширина квазиуровня Г, являясь трудно оцениваемой характеристикой, рассматривается, как правило, в качестве подгоночного параметра [57]. Сделанные здесь оценки Г отвечают максимальным значениям, так что полученные величины $\zeta$ есть оценки по минимуму. Действительно, во-первых, p-состояние атома углерода взаимодействует не с $d$-зоной, а с гибридизованной $s d$-зоной. Плотность состояний $s$-зоны $\rho_{s}$ значительно ниже, чем плотность состояний $d$-зоны $\rho_{d}$ : по оценкам Андерсона для меди $\rho_{s} \approx 0.1 \mathrm{eV}^{-1}[58], \rho_{d} \approx 1 \mathrm{eV}^{-1}$. Во-вторых, имеются сложности при оценке матричного элемента $V$, входящего в выражение (П2) (см. приложение к статье [59]). Так, например, для магнитных примесей в немагнитном металле Андерсон приводит значения $V \approx 2-3 \mathrm{eV}, \Gamma \approx 2-5 \mathrm{eV}$ [58]. С большой степенью вероятности можно, однако, утверждать, что если скачок электронного состояния атомов углерода и релаксация слоя ЭГ и возможны, то только для квазисвободного графена. Отмеченная неопределенность приведенных нами оценок не является, вообще говоря, следствием именно модельного подхода к проблеме, так как результаты, полученные путем численных расчетов, также весьма заметно отличаются (ср., например, $E_{B}$ в табл. 1 и 2).

Итак, в работе получены аналитические выражения для упругой $(k)$ и электрон-фононных $(w, \lambda$ и $\xi)$ констант связи через энергию связи $E_{B}$ графена с подложкой и расстояние $d$ между ними для слабого ван-дерваальсова и сильного кулоновского взаимодействий. Подобного рода оценки представляют интерес при условии, что они приложимы к достаточно широкому кругу объектов. Поэтому здесь рассмотрели как металлические, так и неметаллические подложки. Для удобств дальнейшего использования полученные формулы сведены в табл. 6.

Таблица 6. Формулы для оценки параметров $(N-$ количество зонных состояний)

\begin{tabular}{|c|c|c|c|c|}
\hline Параметры & $k$ & $w$ & $\lambda$ & $\xi$ \\
\hline $\begin{array}{l}\text { Ковалентная } \\
\text { связь металл- } \\
\text { полупроводник }\end{array}$ & $\begin{array}{l}32 E_{B} / d^{2} \\
18 E_{B} / d^{2}\end{array}$ & $\begin{array}{l}4 E_{B} / d \\
9 E_{B} / d\end{array}$ & $\begin{array}{c}E_{B} / 2 \\
9 E_{B} / 2\end{array}$ & $\begin{array}{l}W / 8 \pi N E_{B} \\
2 W / 9 \pi N R_{B}\end{array}$ \\
\hline $\begin{array}{l}\text { Ван-дер-ваальсова } \\
\text { связь }\end{array}$ & $27 E_{B} / d^{2}$ & $9 E_{B} / 2 d$ & $3 E_{B} / 4$ & $W / 3 \pi N E_{B}$ \\
\hline
\end{tabular}




\section{Приложение}

1. Запишем гамильтониан системы графен-подложка в виде следующей суммы [19,29]:

$$
H=H^{e l}+H^{p h}+H^{e l-p h} .
$$

Здесь электронный гамильтониан $H^{e l}=\sum_{i} h_{i}^{e l}$, где

$$
h_{i}^{e l}=\sum_{\mathbf{q}} \varepsilon_{s u b}(\mathbf{q}) \hat{n}_{\mathbf{q}}+\varepsilon_{0} \hat{n}_{i}+V \sum_{\mathbf{q}, i}\left(c_{\mathbf{q}}^{+} a_{i}+h . c\right),
$$

$\varepsilon_{s u b}(\mathbf{q})$ - закон дисперсии электронов подложки, $\varepsilon_{0}-$ энергия $p_{z}$-орбитали атома углерода, $\hat{n}_{\mathbf{q}}=c_{\mathbf{q}}^{+} c_{\mathbf{q}}-$ оператор числа заполнения состояния $|\mathbf{q}\rangle$ с волновым вектором $\mathbf{q}, c_{\mathbf{q}}^{+}\left(c_{\mathbf{q}}\right)$ - соответствующий оператор рождения (уничтожения) электрона подложки, $V$ - матричный элемент взаимодействия, спаривающий состояния $|\mathbf{q}\rangle$ с состоянием $i$-го адатома углерода $|i\rangle$.

Для колебаний листа графена относительно подложки фононный гамильтониан

$$
H^{p h}=\frac{1}{2} M \dot{d}^{2}+\frac{1}{2} k(\delta d)^{2},
$$

где $k$ - силовая (упругая) константа связи графенметалл, $d-$ расстояние графен-подложка, $\delta d-$ вариация длины связи. Здесь мы предположили, что лист графена колеблется относительно подложки как целое.

Гамильтониан электрон-фононной связи графена с подложкой

$$
H^{e l-p h}=\sum_{i} h_{i}^{e l-p h}
$$

где $h_{i}^{e l-p h}=w \delta d \hat{n}_{i}, \quad w$ - деформационная константа электрон-фононного взаимодействия. Легко показать $[19,29]$, что энергия $p_{z}$-орбитали атома углерода $\varepsilon_{0}$ переходит в

$$
\varepsilon(n)=\varepsilon_{0}-\lambda n,
$$

где $\lambda=w^{2} / k$. Здесь мы положили $n_{i}=n$.

2. Учтем полярность связи графен-подложка, для чего в соответствии с МСО [16] вместо выражения (2) запишем $E_{b}=2\left(-\sqrt{V_{2}^{2}+V_{3}^{2}}+V_{\text {rep }}\right)$, где полярная энергия $V_{3}=\left|E_{d}-E_{p}\right| / 2$. Тогда для равновесного значения $d$ найдем $V_{\text {rep }}=\alpha_{c} V_{2} / 2$, где введена ковалентность связи $\alpha_{c}=V_{2} / \sqrt{V_{2}^{2}+V_{3}^{2}}$. В результате получаем $E_{b}=-2\left(V_{2} / \alpha_{c}\right)\left(1-\alpha_{c}^{2} / 2\right), k=32 \alpha_{c}^{3} V_{2} / d^{2}$ и $\lambda=V_{2} / 2 \alpha_{c}^{3}$.

Рассмотрим в качестве подложки $\operatorname{Ir}(111)$. По данным справочника [38] имеем $r_{\mathrm{Ir}}=1.36 \AA$, откуда $d=2.13 \AA$. Так как для иридия $r_{d}=1.08 \AA$ [34] и $r_{p}=6.59 \AA$ [60], получим $V_{2} \approx 2.0 \mathrm{eV}$. Для оценки ковалентности связи примем $E_{d}=7.35 \mathrm{eV}$ и $E_{F}=10.4 \mathrm{eV}$, где энергия отсчитывается от дна зоны проводимости иридия [30]. Так как работа выхода $\operatorname{Ir}(111)$ равна $5.76 \mathrm{eV}$ [38], то энергия $E_{d}=-8.81 \mathrm{eV}$ относительно вакуума. Полагая энергию $E_{p}$ равной энергии ионизации углерода $I=8.30 \mathrm{eV}$ [38], получим $V_{3}=0.26 \mathrm{eV}$, откуда $\alpha_{c} \approx 0.99$.
Аналогичный расчет для металлов, входящих в табл. 1 , дает: $\alpha_{c} \approx 0.86$ для $\mathrm{Co}, \alpha_{c} \approx 0.92$ для $\mathrm{Ni}, \alpha_{c} \approx 0.90$ для $\mathrm{Cu}, \alpha_{c} \approx 0.97$ для $\mathrm{Au}, \alpha_{c} \approx 0.99$ для $\mathrm{Pt}, \alpha_{c} \approx 1$ для $\mathrm{Pd}$ и Ag. Таким образом, для целей настоящей работы поправками на полярность связей вполне можно пренебречь.

3. Рассчитаем полярность связи $p_{z}$-орбитали атома углерода графена с $s p^{3}$-орбиталью атома подложки $\alpha_{c}$. Полярная энергия $V_{3}=\left|\varepsilon_{s p^{3}}(\mathrm{Si})-\varepsilon_{p}(\mathrm{C})\right| / 2$, где $\varepsilon_{s p^{3}}(\mathrm{Si})=\left[\varepsilon_{s}(\mathrm{Si})+3 \varepsilon_{p}(\mathrm{Si})\right] / 4-$ энергия $s p^{3}$-орбитали атома кремния. Воспользовавшись таблицами атомных термов Манна [61], получим $\varepsilon_{s p^{3}}(\mathrm{Si})=-9.39 \mathrm{eV}$, $\varepsilon_{p}(\mathrm{C})=-11.07 \mathrm{eV}$ (отсчет от вакуума), $V_{3}=0.42 \mathrm{eV}$. Так как $V_{2}=2.52 \mathrm{eV}$, имеем $\alpha_{c} \approx 0.99$. Поэтому в формулах работы [32] можно считать $\alpha_{c}=1$, а также пренебречь металличностью связи, полагая $V_{1}=0$.

\section{Список литературы}

[1] J.-C. Charlier, P.C. Eklund, J. Zhu, A.C. Ferrari. In: Carbon nanotubes, topics appl. physics / Eds A. Jorio, G. Dresselhaus, M.S. Dresselhaus. Springer-Verlag, Berlin-Heidelberg, 2008. V. 111. P. 673-709.

[2] L.M. Malard, M.A. Pimenta, G. Dresselhaus, M.S. Dresselhaus. Phys. Rep. 473, 51 (2009).

[3] D. Yoon, H. Cheong. In: Raman spectroscopy for nanomaterials characterization / Ed. S.S.R. Kumar. Springer (2012). P. 191-214.

[4] A.C. Ferrari, D.M. Basko. Nature Nanotechnol. 8, 235 (2013).

[5] A. Politano. Crit. Rev. Solid State Mater. Sci. 42, 99 (2017).

[6] K.C. Haas. Phys. Rev. B 46, 150 (1992).

[7] C. Lobo, J.L. Martins. Z. Phys. D 39, 159 (1997).

[8] K. Ishikawa, T. Ando. J. Phys. Soc. (Jpn) 75, 084713 (2006).

[9] L.A. Falkovsky. ЖЭТФ 132, 446 (2007).

[10] Л.А. Фальковский. ЖЭТФ 142, 560 (2012).

[11] M. Mohr, J. Maultzsch, E. Dobardžić, S. Reich, I. Milošević, M. Damnjanović, A. Bosak, M. Krisch, C. Thomsen. Phys. Rev. B 76, 035439 (2007).

[12] S.V. Kusminskiy, D.K. Campbell, A.H. Castro Neto. Phys. Rev. B 80, 035401 (2009).

[13] T. Aizawa, R. Souda, Y. Ishizawa, H. Hirano, T. Yamada, K. Tanaka. Surf. Sci. 237, 194 (1990).

[14] С.Ю. Давыдов. ФТТ 59, 610 (2017).

[15] S. Ulstrup, M. Bianchi, R. Hatch, D. Guan, A. Baraldi, D. Alfe, L. Hornekær, P. Hofmann. Phys. Rev. B 86, 161402(R) (2012); erratum: Phys. Rev. B 93, 239901(E) (2016).

[16] M. Endlich, A. Molina-Sanchez, L. Wirtz, J. Kröger. Phys. Rev. B 88, 205403 (2013).

[17] J.C. Johannsen, S. Ulstrup, M. Bianchi, R. Hatch, D. Guan, F. Mazzola, L. Hornekær, F. Fromm, C. Raidel, T. Seyller, P. Hofmann. J. Phys.: Condens. Matter 25, 094001 (2013).

[18] T. Sohier. Electrons and phonons in graphene: electronphonon coupling, screening and transport in the field effect setup; Physics [physics]. Universit_e Pierre et Marie Curie - Paris VI, 2015. English. <NNT: 2015PA066393>. $<$ tel-01274687>

[19] С.Ю. Давыдов. ФТТ 55, 197 (2013).

[20] M. Vanin, J.J. Mortensen, A.K. Kelkkanen, J.M. Garcia-Lastra, K.S. Thygesen, K.W. Jacobsen. Phys. Rev. B 81, 081408(R) (2010). 
[21] B. Busse, P. Lazic, R. Djemour, J. Coraux, T. Gerber, N. Atodiresei, V. Caciuc, R. Brako, A.T. N'Diaye, S. Blügel, J. Zegenhagen, T. Michely. Phys. Rev. Lett. 107, 036101 (2011).

[22] I. Hamada, M. Otani. Phys. Rev. B 82, 153412 (2010).

[23] J. Sławinska, P. Dabrowski, I. Zasada. Phys. Rev. B 83, 245429 (2011).

[24] M. Kralj, I. Pletikosić, M. Petrović, P. Pervan, M. Milun, A.T. N’Diaye, C. Busse, T. Michely, J. Fujii, I. Vobornik. Phys. Rev. B 84, 075427 (2011).

[25] A. Dahal, M. Batzill. Nanoscale 6, 2548 (2014).

[26] A. Al Taleb, D. Farias. J. Phys.: Condens. Matter 28, 103005 (2016).

[27] Z.H. Aitken, R. Huang. J. Appl. Phys. 107, 123531 (2010).

[28] K. Zhang, M. Arroyo. J. Appl. Phys. 113, 193501 (2013).

[29] С.Ю. Давыдов. ФТП, 52, 240 (2018).

[30] В.Ю. Ирхин, Ю.П. Ирхин. Электронная структура, физические свойства и корреляционные эффекты в $d$ - и $f$-металлах и их соединениях. Изд-во УрО РАН, Екатеринбург (2004). 472 с.

[31] S. Froyen. Phys. Rev. B 22, 3119 (1980).

[32] С.Ю. Давыдов, О.В. Посредник. ФТТ 57, 819 (2015).

[33] D. Maccariello, D. Campi, A. Al Taleb, G. Benedek, D. Farias, M. Bernasconi, R. Miranda. Carbon 93, 1 (2015).

[34] A. Allard, L. Wirtz. Nano Lett. 10, 4335 (2010).

[35] D. Fariasa, K.H. Rieder, A.M. Shikin, V.K. Adamchuk, T. Tanaka, C. Oshima. Surf. Sci. 454-456, 437 (2000).

[36] W.A. Harrison. Phys. Rev. B 74, 205101 (2006).

[37] У. Харрисон. Электронная структура и свойства твердых тел. Мир, М. (1983). 1, 382 с.; 2, 332 с.

[38] Физические величины. Справочник / Под ред. И.С. Григорьева, Е.3. Мейлихова. Энергоатомиздат, М. (1991). $1232 \mathrm{c}$.

[39] S. Das, D. Lahiri, D.-Y. Lee, A. Agrawal, W. Choi. Carbon 59, 121 (2013).

[40] C. Berger, E.H. Conrad, W.A. de Heer. Epigraphene: epitaxial graphene on silicon carbide. arXive: 1704.00374.

[41] G.H. Wells, T. Hopf, K.V. Vassilevski, E. EscobedoCousin, N.G. Wright, A.B. Horsfall, J.P. Goss, A.G. O'Neill, M.R.C. Hunt. Appl. Phys. Lett. 105, 193109 (2014).

[42] A. Mattausch, O. Pankratov. Phys. Rev. Lett. 99, 076802 (2007).

[43] F. Varchon, R. Feng, J. Hass, X. Li, B.N. Nguyen, C. Naud, P. Mallet, J.-Y. Veuillen, C. Berger, E.H. Conrad, L. Magaud. Phys. Rev. Lett. 99, 126805 (2007).

[44] B. Huang, H.J. Xiang, S.-H. Wei. Phys. Rev. B 83, 161405(R) (2011).

[45] С.Ю. Давыдов, Г.И. Сабирова. Письма в ЖТФ 37, 11, 51 (2011).

[46] G. Persson, U. Lindefelt. J. Appl. Phys. 82, 5496 (1997).

[47] J. Wang, D.C. Sorescu, S. Jeon, A. Belianinov, S.V. Kalinin, A.P. Baddorf, P. Maksymovych. Nature Commun. 7, 13263 (2016).

[48] Z. Zong, C.-L. Chen, M.R. Dokmeci, K-t. Wan. J. Appl. Phys. 107, 026104 (2010).

[49] R. Zhang, C. Zhang, H. Luan, W. Ji, P. Wang. RSC Adv. 5, 35377 (2015).

[50] W. Gao, P. Xiao, G. Henkelman, K.M. Liechti, R. Huang. J. Phys. D: Appl. Phys. 47, 255301 (2014).

[51] K. Kamiya, N. Umezawa, S. Okada. Phys. Rev. B 83, 153413 (2011).

[52] B. Sachs, T.O. Wehling, M.I. Katsnelson, A.I. Lichtenstein. Phys. Rev. B 84, 195414 (2011).
[53] W. Zhou, Y. Liu, Y. Yang, P. Wu. J. Phys. Chem. C 118, 6448 (2014).

[54] С.С. Некрашевич, В.А. Гриценко. ФТТ 56, 209 (2014).

[55] J.C. Garcia, A.T. Lino, L.M.R. Scolfaro, J.R. Leite, V.N. Freire, G.A. Farias, E.F. da Silva jr. 27th Intern. Conf. on the Physics of Semiconductors ICPS-27. AIP Conference Proceedings 772, 189 (2005).

[56] Y.-N. Xu, W.Y. Ching. Phys. Rev. B 44, 7787 (1991).

[57] С.Ю. Давыдов. Теория адсорбции: метод модельных гамильтонианов. Изд-во СПбГЭТУ ЛЭТИ, СПб. (2013). 235 c.; twirpx.com/file/1596114/

[58] Ч. Киттель. Квантовая теория твердых тел. Наука, М. (1967). Гл. 18.

[59] С.Ю. Давыдов. ФТП 46, 204 (2012).

[60] W.A. Harrison, G.K. Straub. Phys. Rev. B 36, 2695 (1987).

[61] W.A. Harrison. Phys. Rev. B 31, 2121 (1985). 